\title{
A Lunar LIGO for NASA's Return to the Moon
}

\author{
Thomas L. Wilson \\ NASA, Johnson Space Center, Houston, TX, USA \\ Email:Thomas.Wilson@cern.ch
}

How to cite this paper: Wilson, T.L. (2021) A Lunar LIGO for NASA's Return to the Moon. Journal of Modern Physics, 12, 536-539.

https://doi.org/10.4236/jmp.2021.124035

Received: December 12, 2020

Accepted: March 19, 2021

Published: March 22, 2021

Copyright (C) 2021 by author(s) and Scientific Research Publishing Inc. This work is licensed under the Creative Commons Attribution International License (CC BY 4.0).

http://creativecommons.org/licenses/by/4.0/

\begin{abstract}
The feasibility of a Lunar LIGO (Laser Interferometer Gravitational-Wave Observatory) was introduced in 1986 as part of NASA's planned return to the Moon by the end of the last century. That return to the Moon mission was cancelled, but is once again planned as Artemis in 2024. In this paper, the feasibility of such a Lunar LIGO as part of NASA's return there will be discussed for that program. Details of the physics of the original Lunar LIGO proposal as a potential portion of future lunar base astronomy and astrophysics designs are presented. Results from NASA's original planned return to the Moon to establish a gravitational wave observatory there are presented and discussed.
\end{abstract}

\section{Keywords}

Gravitational Waves, Astronomy on the Moon, Astrophysics

\section{Introduction}

The conceptual design of NASA's planned Lunar base was addressed at Stanford University in 1989 [1] [2] [3], with a Lunar LIGO (Laser Interferometer Gravitational-Wave Observatory) first discussed in [3]. The present author organized the Stanford workshop and its focus on gravitational radiation, and pursued the Lunar LIGO concept for years thereafter [4]-[10] while serving as lead for physics and astrophysics mission development in the Solar System Exploration Division at NASA's Johnson Space Center. That lunar return effort continued until it was terminated for lack of funding in 1998.

The subsequent work was presented in various ways [4]-[10] while the Earth-based LIGO concepts were undergoing initial development. The latter obviously have come to marvelous fruition in the quarter century since, with the advent of Advanced LIGO and Advanced VIRGO systems [11] [12] [13] in the LIGO and LISA [14] Scientific Collaborations (LSC) established in 1997. In- 
cluded are their marvelous discoveries in Earth-based gravitational wave astronomy, not to mention the discovery of gravitational waves per se [16].

The question presented here is the virtue of adding a Lunar LIGO to these conceptual configurations [3]-[16], and how that can augment the Earth-based LIGO systems. The result is a superior gravitational wave measurement system for examining gravitational waves and their astrophysical sources in the solar system and surrounding galaxies. This is not available using the Earth-based systems alone.

\section{Why Lunar LIGO?}

The preliminary proposal [8] [9] that the Earth-based multi-LIGO system can be augmented with a Lunar LIGO appears promising. It consists of emplacing a modest LIGO optical system on the Moon, proving to be a simple and advantageous application in the vacuum environment of the lunar surface. Less seismic, gravity gradient, tidal, and acoustic noise will yield greater sensitivity at frequencies between 0.25 and $3 \mathrm{~Hz}$ on the lunar surface.

Emplacement can be accomplished using unmanned robotic landers such as the Artemis project or by any manned landing program.

Mechanical decoupling from the Earth-based antennas will yield a method of noise filtering (coincidence with terrestrial antennas over their common frequencies) and provide a significant sanity check on wave versus pulse events.

Operating along with Earth-based antennas, it will provide a longer baseline for the localization of gravitational wave sources. This gives 50 times better angular resolution than the Earth-based antennas alone. Also the lunar antenna will not be mechanically or geophysically coupled to the terrestrial antennas, thus providing a significant confidence or voting factor for detected events.

The distance between the Earth and Moon provides a long parallax baseline with terrestrial antennas for locating the sources of a gravitational event. Given that the lunar vacuum eliminates the need for long evacuated tunnels, a minimal Fabry-Perot antenna could be placed on the Moon using three robotic landers, one containing the laser source, the beam splitter, the detector, recycle mirrors, cavity mirrors, and other optics. The other two landers would contain the end mirrors of the interferometer arms, and provide closure phase along the third leg.

The lack of arm enclosures allows the arms to be extremely long, limited only by a lunar radius of $1738 \mathrm{~km}$. Appropriate choice of landing sites could allow for longer arms by taking advantage of local topography. One can easily adjust the length of the arms by moving the landers containing the end mirrors [7]. The entire Moon can also be visualized as a gravitational detector itself [15] by configuring it with an array of seismometers.

The lunar environment will likewise provide significant advantages. It will eliminate the need to maintain a vacuum in the interferometer arms over the life-cycle of the antenna. This, coupled with the lack of construction costs 
needed to enclose and pull a vacuum in the arms, should offset much or all of the cost of launch and delivery. Lesser surface gravity will prove better for mechanical isolation of optics [6].

Obviously, there are distinct advantages through which Lunar LIGO and LISA Pathfinder programs can mutually overlap and supplement one another. One might even begin by transporting other configurations to the lunar base to become a lunar LIGO.

An advanced, man-tended version of the Lunar LIGO would allow for even more flexibility (for instance Figure 1, adapted from [8]). Detectors and mirrors could be repaired and/or upgraded by the lunar base personnel. The antenna could be actively monitored and seismic data could be screened using gravimeters to aid in the data's noise analysis. For instance, a large array of antennas could be built to allow for better spurious signal elimination by coincidence [7]. A large number of antennas would be easier to build and maintain on the Moon than on the Earth due to the lack of evacuated tunnels.

\section{Conclusions}

In conclusion, this investigation shows that a lunar-based Fabry-Perot gravitational wave antenna would provide a valuable complement to Earth-based systems, both for conclusive first detection and for continued gravitational wave astronomy there. Furthermore, due to unique features of the lunar environment, the life-cycle costs could be competitive with Earth-based antennas.

This investigation further shows that a lunar-based Fabry-Perot gravitational wave antenna would provide a valuable complement to the Earth-based systems, both for conclusive, first detection and for continued gravitational wave astronomy. Furthermore, due to unique features of the lunar environment, the life-cycle costs could be competitive with Earth-based antenna systems.

After decades of studying the elusive phenomena predicted by Einstein's general

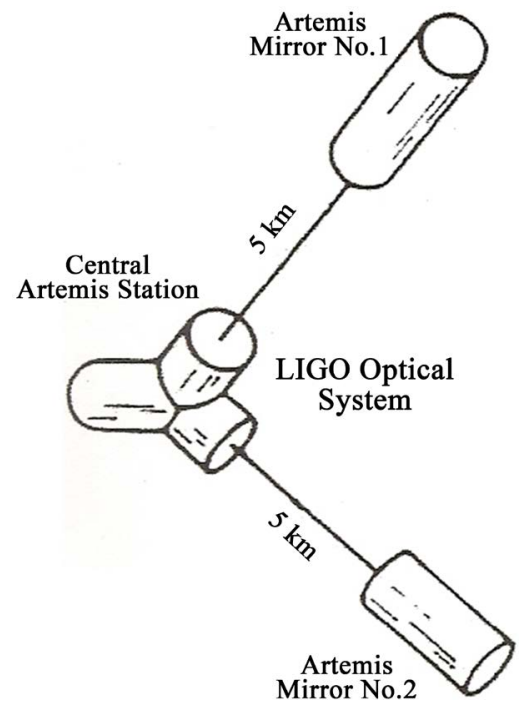

Figure 1. Lunar LIGO using Artemis. 
theory of relativity, gravitational radiation has been discovered [16]. It is important that such research be continued, and one way to do so is to expand our notions of the Moon to serve as another platform for enhancing these investigations.

In closing, other proposals have also been developed, which include DECIGO, GLOC [15] [17] [18].

This very preliminary proposal that the Earth-based multi-LIGO system can be supplemented by a lunar LIGO system appears promising.

\section{Conflicts of Interest}

The author declares no conflicts of interest regarding the publication of this paper.

\section{References}

[1] Potter, A. and Wilson, T.L. (1989) Physics \& Astrophysics from a Lunar Base. AIP Conference Proceedings, No. 202, American Institute of Physics, New York.

[2] Weber, J. (1990) AIP Conference Proceedings, 202, 159-202. https://doi.org/10.1063/1.39128

[3] Stebbins, R.T. and Bender, P.L. (1990) AIP Conference Proceedings, 202, 188-201. https://doi.org/10.1063/1.39103

[4] Wilson, T.L., Blome, H.J. and LaFave, N. (1996) Space V, Proc. of Fifth Int'l. Conf. on Space 96, 2, 861-863.

[5] LaFave, N. and Wilson, T.L. (1995) Gravitational Wave Astronomy from the Moon. Lunar and Planetary Science Conference, Houston, 13-17 March 1995, 817-818.

[6] Wilson, T. and LaFave, N. (1994) Astronomy Session: Gravitational Wave Astronomy \& the Lunar LIGO Concept. Space IV, Vol. 2, 1442-1451.

[7] Wilson, T.L. and LaFave, N. (1994) Lunar LIGO \& Gravitational Wave Astronomy on the Moon. Lunar \& Planetary Science Conference, Vol. 3, Houston, 1499.

[8] LaFave, N. and Wilson, T.L. (1993) Lunar LIGO: A New Concept in Gravitational Wave Astronomy. Lunar \& Planetary Science Conference, Houston, 15-19 March 1993, 841.

[9] LaFave, N. and Wilson, T.L. (1992) Astronomy on the Moon. Lunar \& Planetary Science Conference, Houston, 16-20 March 1992, 751.

[10] Wilson, T., et al. (2005) Gravitational Wave Astronomy on the Moon. Proceedings of the Space Nuclear Conference, San Diego, 5-9 June 2005, Paper 1158.

[11] Abramovici, A., et al. (1992) Science, 256, 325. https://doi.org/10.1126/science.256.5055.325

[12] LIGO Details Can Be Found at ligo.caltech.edu.

[13] VIRGO Is a Large European Michelson Interferometer Operating in Italy.

[14] LISA (Laser Interferometer Space Antenna) Is the First Proposed Orbital Space-Based Gravitational Wave Antenna. See lisa.nasa.gov.

[15] DECIGO (DECi-hertz Interferometer Grav. Wave Observatory).

[16] Abbott, B.P., et al. (2016) Physical Review Letters, 116, Article ID: 061102. https://doi.org/10.1103/PhysRevLett.116.061102

[17] GLOC, Gravitational-Wave Lunar Observatory for Cosmology.

[18] Johnson, W.W. (1989) AIP Conference Proceedings, 202, 183-187. 